\title{
ON A CLASS OF FINITELY DETERMINED PLANAR DOMAINS
}

\section{Mihai Putinar}

\section{Introduction}

This note describes a class of planar domains which are determined by finitely many of their moments. A classical result whose proof can be traced back to the last century to the works of P. L. Cebyshov and A. A. Markov asserts that the only sets of the real axis which are finitely determined by their moments are the unions of finite intervals. It was M. G. Krein who has continued the theory of Cebyshov and Markov during this century, by developing its functional analytic roots and by finding a wide range of applications. The monographs [2], [14] and the survey paper [13] treat these questions in detail.

The domains discussed in the present note are known in literature as "quadrature domains" and they appear in at least four different contexts: exact quadrature formulae for analytic functions, the inward balayage phenomenon in potential theory, free boundary problems for partial differential equations and the Schwarz function and conformal maps derived from it. The monographs [9] and [21] are devoted to these topics.

Let $F$ be a bounded closed set of the complex plane. The moments of $F$ are the complex numbers:

$$
a_{m n}=\int_{F} z^{m} \bar{z}^{n} d A(z), \quad m, n \in N,
$$

where $d A$ stands for the Lebesgue planar measure. Together with the double sequence of moments it is natural to consider (following the work of Markov and that of Akhiezer and Krein in one variable) the following exponential transform:

$$
\sum_{m, n=0}^{\infty} b_{m n} X^{m+1} Y^{n+1}=1-\exp \left(-\sum_{m, n=0}^{\infty} a_{m n} X^{m+1} Y^{n+1}\right) .
$$

The latter indentity is considered in the formal series ring with two independent variables $X, Y$.

Received January 21, 1994

Paper supported by a grant from the National Science Foundation 
We call the moment sequence $a_{m n}$ degenerated if $\operatorname{det}\left(b_{m n}\right)_{m, n=0}^{N}=0$ for a finite positive integer $N$. The set $F$ is in that case determined by the finite sequence $a_{m n}, 0 \leq m, n \leq N$, and it corresponds exactly to the union of intervals in the one variable theory.(See [2]). The structure of these sets supporting degenerated moment sequences is expected to be quite rigid. The first result along these lines can be stated as follows.

Theorem 1. The compact closed set $F \subset C$ has a degenerated moment sequence if and only if it is bounded by real algebraic curves and there is a meromorphic function in int $(F)$ which extends continuously the function $\bar{z}$ from the boundary of $F$.

Since the set $F$ satisfying the condition stated in Theorem 1 has piecewise smooth boundary, we can equally work with its interior, which has the same moments. The natural conformal map from a circular slit domain onto $\operatorname{int}(F)$ reveals partially the geometry and the analytic structure of these supports of degenerated moment sequences.

Theorem 2. Let $\Omega=\operatorname{int}(F)$, where $F$ is a compact set with a degenerated moment sequence, and let $\Delta$ be a connected component of $\Omega$.

a). $\Delta$ is simply connected if and only if there is a rational conformal map from the unit disk onto $\Delta$;

b). $\Delta$ is one-connected if and only if it is the image of the rectangle $R=$ $\{z \in C ; 0<\Re z<a, 0<\Im z<2 b\}$ by an elliptic function with periods $2 a, 2 b i$ and which is one to one on $R$.

In general, for a multiply connected domain $\Delta$ the conformal map from a circular slit domain $D$ onto $\Delta$ extends to a meromorphic function defined on a compact Riemann surface which doubles $D$ in a canonical way.

The proof of Theorem 2 is based on a repeated application of the symmetry principle to the meromorphic function which extends $\bar{z}$ from the boundary of $F$. Once we observe that the domains described in Theorem 1 are quadrature domains ( in a sense which will be explained in Section 4 below), the first part of Theorem 2 is not new, see for references [1] and [9]. Moreover, Gustafsson has proved in [11] that in any conformal class of a finitely connected domain there is at least one representative which fulfils the condition in Theorem 1. The geometry of these (quadrature) domains is remarkable, and it is still investigated at present by several authors, (see the references in [21]).

In the proof of Theorem 1 three objects, naturally related with each other and representing different interpretations of the moment sequence $a_{m n}$, appear. The next result enlightens this relationship. To simplify the 
statements we adopt the following notation, for a given compact set $F$ and a point $z \in C \backslash F$ :

$$
E(F)(z)=\exp \left(\frac{-1}{\pi} \int_{F} \frac{d A(\zeta)}{|\zeta-z|^{2}}\right)
$$

Theorem 3. Let $F$ be a compact planar set. The following conditions are equivalent:

a). F has a degenerated moment sequence;

b). The function $E(F)(z)$ is rational for $|z|$ large, with a denominator of the form $|p(z)|^{2}$, where $p$ is a polynomial;

c). There is a bounded linear operator $T$ acting on a Hilbert space, with spectrum equal to $F$, with rank one self commutator and satisfying $p(T)^{*}\left[T^{*}, T\right]=0$, for a polynomial $p$.

The question treated in this note ( that of characterizing the sets with degenerated moment sequences ) is a part of a more general problem, related to arbitrary functions bounded by 0 and $L$ - the so called $L$-problem of moments. The operator theoretic aspects of this problem were discussed in $[19,20]$. A very general conclusion of these papers is that the inverse problem for the principal function of a hyponormal operator with rankone self-commutator is equivalent to the $L$-problem of moments. This explains the presence of the function $E(F)$ (a two variable perturbation determinant) in the statement of Theorem 3.

The class of Hilbert space operators with one dimensional self-commutator is very well understood by now, thanks to the works of Pincus [17], Carey and Pincus [5], Helton and Howe [12], and more recently Clancey [8], Pincus, J. Xia and D. Xia [18] and several other authors. A unitary invariant for these operators is a measurable function with values in the interval $[0,1]$, which extends naturally the Fredholm index across the spectrum. This function was called the principal function in [17], and it was the subject of many investigations since then. The monographs $[8,15,22]$ are devoted to this subject.

The proof of Theorem 1 makes use of the theory of the principal function and the properties of the kernel $E(F)$, which plays the role of a characteristic function for the class of operators with rank one self-commutator. It must be noted that these objects are two dimensional generalizations of the phase shift, perturbation determinant and trace formulae, all due to M. G. Krein.

The next section recalls the dictionary between the $L$-problem of moments, operators with rank one self-commutator and the kernels $E(F)(z)$. Section 3 contains a few steps in the proofs of the main results. In the 
last section we briefly discuss the relationship between the domains which carry degenerated moments and quadrature domains.

\section{The $L$-problem of moments}

Let $T$ be a bounded linear operator acting on a complex Hilbert space $H$, having one dimensional self-commutator:

$$
\left[T^{*}, T\right]=\xi \otimes \xi
$$

(In some respect, this is the class of operators closest to the normal operators.) We call $T$ pure if there is no normal operator which is a direct orthogonal summand of $T$. Let $z, w$ be points in the resolvent set of $T$. The following multiplicative commutator plays an important role in the parametrization of the operators $T$.

$$
\begin{aligned}
C(z, w) & =(T-z)^{*-1}(T-w)(T-z)^{*}(T-w)^{-1} \\
& =I-(T-z)^{*-1}\left[T^{*}, T\right](T-w)^{-1} \\
& =1-\left\langle(T-z)^{*-1} \xi,(T-w)^{*-1} \xi\right\rangle .
\end{aligned}
$$

A generalization of Krein's determinantal formula from the perturbation theory of self-adjoint operators (formulated for the first time in [17] ) asserts that there is a measurable function $g: C \longrightarrow[0,1]$, supported by the spectrum of $T$ and characterized by the following identity:

$$
\operatorname{det} C(z, w)=\exp \left(\frac{-1}{\pi} \int_{C} \frac{g(\zeta) d A(\zeta)}{(\bar{\zeta}-\bar{z})(\zeta-w)}\right)
$$

which is valid for any points $z, w$ in the resolvent set of $T$.

The function $g$ is called the principal function of the operator $T$ and it encodes the spectral behaviour of the pure part of $T$. In fact there is a bijection between pure Hilbert space operators with rank one nonnegative self-commutator and the set of elements $g \in L^{1}{ }_{\text {comp }}(C)$ satisfying $0 \leq g \leq 1$, a.e. We mention that there are at least three different ways of constructing the operator $T$ from the principal function $g$. (See $[8,15,18,23])$.

Based on the equivalence between the above class of operators and their principal functions we can easily approach the $L$-problem of moments. Let us consider the moments of a virtual principal function:

$$
a_{m n}=\int_{C} z^{m} \bar{z}^{n} g(z) d A(z), \quad m, n \in N .
$$


Then the exponential transform:

$$
1-\exp \left(\frac{-1}{\pi} \sum_{m, n=0}^{\infty} a_{m n} X^{m+1} Y^{n+1}\right)=\sum_{m, n=0}^{\infty} b_{m n} X^{m+1} Y^{n+1}
$$

produces from the moments $a_{m n}$ the double sequence:

$$
b_{m n}=\left\langle T^{* n} \xi, T^{* m} \xi\right\rangle
$$

Define further the kernel $K: N^{2} \times N^{2} \longrightarrow C$ using the following rules inductively:

a) $K(0,0 ; m, n)=K(0, m ; 0, n)=b_{m n}$,

b) $K(m, n ; p, q)=\overline{K(p, q ; m, n)}$

c) $K(m+1, n ; p, q)=K(m, n ; p, q+1)+\sum_{r=1}^{m} K(m, n ; r-1,0) b_{p-r, q}$.

Then, similarly to the one dimensional case treated by Akhiezer and Krein [1], one proves that the problem (2) has a solution $g$ with support in the ball centered at the origin with radius $r$ if and only if the kernels $K(m, n ; p, q)$ and $r^{2} K(m, n ; p, q)-K(m+1, n ; p+1, q)$ are non-negative definite. (See [19] for details).

By using properties of the principal function one proves that the solution $g$ of the problem (2) is integer valued whenever there is a polynomial $P$ in two variables with the property that $P\left(T^{*}, T\right) \xi=0$. Here we arrange by convention all powers of $T^{*}$ to be to the left of the powers of $T$. In particular, if there is an integer $N$ with the property that $\operatorname{det}\left(b_{m n}\right)_{m, n=0}^{N}=$ 0 , then the vectors $\xi, T^{*} \xi, \ldots, T^{* N} \xi$ are linearly dependent and hence $g$ is the characteristic function of a compact set $F \subset C$.

According to the identity (1), if we start with $g$ equal to the characteristic function of a compact set $F$, for large values of $|z|$ we obtain the relation:

$$
E(F)(z)=1-\left\|(T-z)^{*-1} \xi\right\|^{2} .
$$

Therefore, assuming that $E(F)(z)=q(\bar{z}, z) /|p(z)|^{2}$, where $q, p$ are polynomials, one easily proves that $p(T)^{*} \xi=0$.

These considerations explain Theorem 3 in the introduction.

We end this section by recalling that the identity (1) was extended in [7] and [8] over the whole complex plane, in a weak sense. In the same papers a series of remarkable properties of the non-negative kernel $\operatorname{det} C(z, w)$ were established. 


\section{The geometry of finitely determined domains}

In view of Theorem 3 , it is equivalent to consider either a compact set $F$ which has a degenerated sequence of moments, or a pure operator $T$ with rank one self commutator $\left[T^{*}, T\right]=\xi \otimes \xi$, subject to the condition $p(T)^{*} \xi=0$, where $p$ is a polynomial.

In this case the function $E(F)(z)$ is rational in $z$ and $\bar{z}$ in a neighbourhood of infinity. By taking the logarithmic derivative of $E(F)(z)$ in $z$ one finds that the function $\int_{F}(\zeta-z)^{-2}(\bar{\zeta}-\bar{z}) d A(\zeta)$ is also rational. A simple argument shows then that the set $F$ is semi-algebraic. (See [20], Lemma 5.2 for details). Thus, one can assume without loss of generality that $F$ is the closure of a bounded open set with real algebraic boundary $F=\bar{\Omega}$. Let $\Omega_{j}, 1 \leq j \leq r$, be the connected components of $\Omega$. Since

$$
E(\Omega)(z)=\prod_{j=1}^{r} E\left(\Omega_{j}\right)(z),
$$

and $E\left(\Omega_{j}\right)(z)<1$ for $z \in C \backslash \bar{\Omega}$, one finds that each of the factors $E\left(\Omega_{j}\right)(z)$ is rational at infinity, with $|p(z)|^{2}$ as denominator. This proves that if the set $\Omega$ has degenerated moments, then each connected component $\Omega_{j}$ has the same property.

Let $\Omega$ be a bounded planar domain with a degenerated sequence of moments. Let $T$ be the associated operator with rank one self-commutator and let $p$ be a polynomial with the property $p(T)^{*}\left[T^{*}, T\right]=0$. The identity (1) gives in particular:

$$
\begin{aligned}
f(z) & =\int_{\partial \Omega} \frac{p(\zeta) \bar{\zeta} d \zeta}{\zeta-z} \\
& =\int_{\partial \Omega} \frac{(p(\zeta)-p(z)) \bar{\zeta} d \zeta}{\zeta-z}+p(z)\left\langle(T-z)^{*-1} \xi, \xi\right\rangle .
\end{aligned}
$$

Hence the function $f(z)$ coincides with a polynomial function $P(z)$ in the complement of $\bar{\Omega}$. Consequently, the Sohotskii-Plemelj-Privalov formula shows that the function $p(z) \bar{z}-P(z)$ extends analytically from $\partial \Omega$ to $\Omega$. This is the main observation which proves half of Theorem 1 .

If $\bar{z}$ extends meromorphically from $\partial \Omega$ to $\Omega$, then one evaluates directly by using the Cauchy formula the kernel $E(\Omega)(z)$ and one proves that it is rational at infinity, with denominator $|p(z)|^{2}$. This part of the proof of Theorem 1 relies also on general properties of the kernel $E(\Omega)$ as derived from the spectral theory of the operator $T$.

Theorem 2 is a consequence of Theorem 1 and the symmetry principle for analytic functions. It is worth mentioning that for a simply connected 
bounded domain $\Omega$, the function $E(\Omega)$ is rational at infinity (without any assumption on the denominator) if and only if the domain $\Omega$ carries a degenerated sequence of moments.

Examples. The simplest example of a bounded domain $\Omega$ as above is a disk $D_{a}(r)$ centered at $a$ and of radius $r$. In that case one easily computes the associated kernel:

$$
E\left(D_{a}(r)\right)(z)=1-\frac{r^{2}}{|z-a|^{2}}, \quad|z-a|>r .
$$

The corresponding operator is in that case $T=r(S-a)$, where $S$ denotes the unilateral shift of multiplicity one. We note that only in that case $T$ is a subnormal operator. Thus the disk $D_{a}(r)$ is determined by four of its moments:

$$
\begin{aligned}
& a_{00}=\pi r^{2} \\
& a_{01}=\overline{a_{10}}=\pi a r^{2} \\
& a_{11}=\pi\left(\frac{r^{4}}{2}+|a|^{2} r^{2}\right)
\end{aligned}
$$

Similarly, a disjoint union of $N$ open disks is determined by the moments $a_{m n}, 0 \leq m, n \leq N$.

It is interesting to remark that the union of two non-disjoint disks (not any contained one in the other) is not finitely determined by its moments. Indeed, in that case the function $\bar{z}$ extends to different meromorphic functions, from different portions of the boundary. This argument also shows that a polygon with straight sides is never finitely determined by its moments.

On the other hand, according to Theorem 2, the Joukovski profile (of an airplane wing) is determined by finitely many of its moments. To be more specific, the image $J$ by the map $z+z^{-1}$ of a disk, not containing the origin in its closure, is determined by the moments $a_{m n}, 0 \leq m, n \leq 2$. Other simple examples of similar domains can be constructed using Theorem 2 .

Several similar examples are contained in [9]. We point out that there is no example of a multiply connected domain which fulfills the condition in Theorem 1. All results about these domains are qualitative and not constructive, cf. [11].

By comparing the results in this note with the classical theory in one variable we expect that the classification of the degenerated moment sequences given in Theorem 1 will enlighten the truncated $L$-problem of moments (2). First it should be pointed out that, contrary to the classical situation, one can prove that in two variables the power moment problem and the $L$-problem are no more in a natural correspondence. However, 
the finite determinacy in the truncated $L$-problem seems to have further analytical and geometrical consequences. Some facts in this direction, as well as the complete proofs of the above results, will appear in a separate paper.

\section{Quadrature domains}

Let $\Omega$ be a bounded domain with piece-wise smooth boundary in the complex plane. We call $\Omega$ a quadrature domain if there is a distribution $u$ supported by finitely many points of $\Omega$, with the property that:

$$
\int_{\Omega} f(z) d A(z)=u(f)
$$

for any analytic and Lebesgue integrable function $f$ in $\Omega$.

It was Phillip Davis (cf. [9] and the references there) who has investigated first these domains, from the point of view of approximation theory and the theory of the Schwarz function. Later, Aharonov and Shapiro [1] have rediscovered these domains in connection with an inverse problem in potential theory. Afterwards, this subject has flourished in several directions. The recent monograph [21] contains many remarks and updated references related to quadrature domains.

The contents of this note adds a new interpretation, based on Hilbert space operators, to the theory of quadrature domains. Next we confine ourselves to explain formula (3) from the point of view of operator theory.

Let $T$ be a bounded linear operator with rank one self-commutator $\left[T^{*}, T\right]=\xi \otimes \xi$, acting on the Hilbert space $H$ and with the property that the space $K$ generated by the vectors $\xi, T^{*} \xi, T^{* 2} \xi, \ldots$ is finite dimensional. Then we know from Theorem 3 that the spectrum of $T$ is the closure of a domain $\Omega$, characterized by the analytic condition in Theorem 1. By using the trace formula derived from the identity (1) we obtain:

$$
\begin{aligned}
\frac{1}{\pi} \int_{\Omega} f(\bar{z}) d A(z) & =\operatorname{Tr}\left[T^{*}, T f\left(T^{*}\right)\right]=\operatorname{Tr}\left[T^{*}, T\right] f\left(T^{*}\right) \\
& =\left\langle f\left(T^{*}\right) \xi, \xi\right\rangle=\left\langle f\left(T^{*} \mid K\right) \xi, \xi\right\rangle
\end{aligned}
$$

where $f$ is an antianalytic function in a neighbourhood of $\bar{\Omega}$. It is obvious that the last expression above represents a distribution with finite support in $\Omega$, applied to the analytic function $f(\bar{z})$. In order to extend this formula to any integrable analytic function in $\Omega$ one can rely on the functional model of $T$ developed in [15] Chapter XI. 
This matricial representation of the quadrature formula (3) has a series of applications, both to the theory of quadrature domains and to the theory of the principal function. We will resume these topics in a separate paper.

\section{References}

1. D. Aharonov and H. S. Shapiro, Domains on which analytic functions satisfy quadrature identities. J. Anal. Math. 30 (1976), 39-73.

2. N. I. Akhiezer and M. G. Krein, Some Questions in the Theory of Moments, Transl. Math. Monographs 2, Amer. Math. Soc., Providence, RI, 1962.

3. L. Brown, The determinant invariant for operators with trace class selfcommutator. in Proc. Conf. Operator Theory, Lecture Notes in Math. 345, Springer, Berlin, 1973.

4. R. W. Carey and J. D. Pincus, An exponential formula for determining functions. Indiana Univ. Math. J. 23 (1974), 1031-1042.

5. _ Commutators, symbols and determining functions. J. Funct. Analysis 19 (1975), 50-80.

6. K. Clancey, Seminormal operators, in Lecture Notes in Math. 742, Springer, Berlin, 1979.

7. _ A kernel for operators with one-dimensional self-commutator. Integral Eq. Operator Theory 7 (1984), 441-458.

8. Theory 13 (1985), 265-289.

9. P. Davis The Schwarz function and its applications. Carus Math. Mono. 17 Math. Assoc. Amer., 1974.

10. B. Fuglede, The multidimensional moment problem. Expo. Math. 1 (1983), 47-65.

11. B. Gustafsson, Quadrature identities and the Schottky double. Acta Appl. Math. 1 (1983), 209-240.

12. J. W. Helton and R. Howe, Trace of commutators of integral operators. Acta Math. 135 (1975), 271-305.

13. M. G. Krein, The ideas of P. L. Cebyshov and A. A. Markov in the theory of limiting values of integrals and their further developments (in Russian). Uspehi Mat. Nauk. 6 (1951), 3-120.

14. M. G. Krein and A. A. Nudelman, Markov moment problem and extremal problems. Transl. Math. Monographs 50, Amer. Math. Soc., Providence, RI, 1977.

15. M. Martin and M. Putinar, Lectures on hyponormal operators, Birkhauser, Basel, 1989.

16. Z. Nehari, Conformal Mapping, McGraw-Hill Co., New York, 1952.

17. J. D. Pincus, Commutators and systems of singular integral equations. Acta Math 121 (1968), 219-249.

18. J. D. Pincus, J. Xia and D. Xia, The analytic model of a hyponormal operator with rank one self-commutator. Integral Eq. Operator Theory 7 (1984), 516-535.

19. M. Putinar, A two-dimensional moment problem. J. Funct. Analysis 80 (1988), $1-8$.

20. The L problem of moments in two dimensions. J. Funct. Analysis 94 (1990), 288-307.

21. H. S. Shapiro The Schwarz function and its generalization to higher dimensions. Univ. Arkansas Lect. Notes 9, John Wiley and Sons, New York, 1992. 
22. D. Xia, Spectral theory of hyponormal operators, Birkhauser, Basel, 1983.

23. Eq. Operator Theory 6 (1983), 444-452.

Department of Mathematics, University of California, Riverside, CA-92521.

E-mail address: mputinar@ucrmath.ucr.edu 